\title{
Wettability transition of plasma-treated polystyrene micro/nano pillars-aligned patterns
}

\author{
J. Kong ${ }^{1,2 *}$, K. L. Yung 2 , Y. X ${ }^{2}$, W. Tian ${ }^{1}$ \\ ${ }^{1}$ Department of Applied Chemistry, School of Science, Northwestern Polytechnical University, Xi' an, 710072, \\ P. R. China \\ ${ }^{2}$ Department of Industrial and Systems Engineering, The Hong Kong Polytechnic University, Hung Hom, Kowloon, \\ Hong Kong
}

Received 30 May 2010; accepted in revised form 17 August 2010

\begin{abstract}
This paper reports the wettability transition of plasma-treated polystyrene (PS) micro/nano pillars-aligned patterns. The micro/nano pillars were prepared using hot embossing on silicon microporous template and alumina nanoporous template, which were fabricated by ultraviolet (UV) lithography and inductive coupled plasma (ICP) etching, and two-step anodic oxidation, respectively. The results indicate that the combination of micro/nano patterning and plasma irradiation can easily regulate wettabilities of PS surfaces, i.e. from hydrophilicity to hydrophobicity, or from hydrophobicity to superhydrophilicity. During the wettability transition from hydrophobicity to hydrophilicity there is only mild hydrophilicity loss. After plasma irradiation, moreover, the wettability of PS micro/nano pillars-aligned patterns is more stable than that of flat PS surfaces. The observed wettability transition and wettability stability of PS micro/nano pillars-aligned patterns are new phenomena, which may have potential in creating programmable functional polymer surfaces.
\end{abstract}

Keywords: nanomaterials, patterns, nanopillars, wettability

\section{Introduction}

Controlling the wettability of a surface has gained huge attention because wettability (hydrophobic, amphiphobic, and hydrophilic etc.) play a crucial role in many natural and industrial applications, e.g. self-cleaning, water repellency, superhydrophobic coatings, intelligent textiles, infection of medical polymers and particle attraction in deoxyribonucleic acid (DNA) purification [1-6]. A surface is hydrophilic when the water contact angle (CA) is lower than $90^{\circ}$ and even considered as superhydrophilic when its $\mathrm{CA}$ is below $5^{\circ}$. On the other hand, the surface is hydrophobic when its CA is greater than $90^{\circ}$. Moreover, the surface becomes superhydrophobic when its CA is above $150^{\circ}$. Generally, the wettability of a solid surface is mainly caused by the chemical composition and structure asperities, which depend on surface energy and geometric structure [7].

To obtain hydrophobic polymer surfaces, coating with low surface energy materials such as fluoroalkylsilane can be helpful [8-12]. On the other hand, geometric structures including rough structures and regular micro/nano patterns are also crucial $[13,14]$. To obtain polymer regular micro/ nano geometric surfaces, various nanoprocessing approaches can be employed, including nanoporous template wetting [15], capillary lithography [16], nanoimprint lithography [17], microstereolithography [18], template rolling press [19], and water spreading of carbon nanotubes [20]. A number of theoretical and experimental studies indicated that 
polymer aligned nanopillar arrays or carbon nanotube arrays could exhibit hydrophobic or surperhydrophobic feature [21-24].

As important as enhancing hydrophobicity of polymer surfaces, controlling hydrophilicity of polymer surfaces is also an interesting issue because most polymers are hydrophobic in nature that makes them unsuitable for important biomedical and industrial applications [25]. Several surface modification techniques have been developed in order to induce polymer hydrophilic surfaces and to preserve desirable bulk properties at the same time. Wet chemical processing, plasma treatment (glow discharge, corona, and flame), and radiation treatment (ultraviolet irradiation and laser treatment) are the main surface modification techniques employed [26]. In particular, plasma treatment of polymeric materials has become one of the most important methods to enhance wettability, printability, and adhesion without affecting bulk material feature $[27,28]$. However the hydrophilicity is often lost when the plasma treated surface is exposed to air again. So, one challenge lies in how to produce superhydrophilic surfaces without hydrophilicity loss or with low hydrophilicity loss during the exposure to air.

In this paper, we report the wettability transition behavior of plasma-treated polystyrene (PS) micro/nano pillars-aligned patterns. The interesting phenomena are that the combination of micro/nano patterning and plasma irradiation can easily regulate PS surfaces' wettabilities, i.e. from hydrophilicity to hydrophobicity, or from hydrophobicity to superhydrophilicity. When wettability transition from hydrophobicity to hydrophilicity occurs, there is only mild hydrophilicity loss. After plasma irradiation, moreover, the wettability of PS micro/nano pillars-aligned patterns is more stable than that of flat PS surfaces fixed with other processing conditions.

\section{Experimental}

\subsection{Materials}

PS was purchased from Sigma-Aldrich Co. (430102, average $M_{w} \sim 192000$, Germany). Micro porous silicon templates with pore diameters of 5 , 10 and $20 \mu \mathrm{m}$ were fabricated using ultraviolet (UV) lithography and subsequent inductive cou- pled plasma (ICP) etching in our laboratory. Nano porous anodic alumina (PAA) template with average pore diameters of $250 \mathrm{~nm}$ was purchased directly (Anodisc 13, Whatman International Ltd, Maidstone, UK). It is through-hole, freestanding disk with channel length of about $50 \mu \mathrm{m}$. PAA template with pore diameters of $100 \mathrm{~nm}$ was prepared by us [29]. Analytic grade chemical reagents including $\mathrm{NaOH}$, ethanol and deionized water were purchased from Alfa Aesar China Co. Ltd (Tianjin, China).

\subsection{Preparation of micro porous silicon template}

To fabricate micro porous silicon template, the combined process of UV lithography and ICP etching were employed [30]. Firstly, the silicon wafer was spin coated using photoresist (BP212) and softly baked under $110^{\circ} \mathrm{C}$ for $1 \mathrm{~min}$ on a Spin Coater (CEE model 100CB, Brewer Science Co., USA). Then UV lithography processing was conducted with a deep UV Mask aligner (MA6, Suss Co., Germany). Then the designed micro porous silicon templates $(5,10,20 \mu \mathrm{m})$ were obtained on the ICP machine (Multiplex Systems ASE, STS Co., UK).

\subsection{Preparation of PS micropatterns and nanopatterns}

The PS micro/nano pillars-aligned patterns were prepared using hot embossing. PS film with a thickness of $1.5 \mathrm{~mm}$ was put on the top of micro porous silicon template or nano porous anodic alumina template. After the machine mould cavity is closed, the temperature was raised to $130^{\circ} \mathrm{C}$. Then a pressure of $0.3 \mathrm{MPa}$ was applied through a hydraulic press to compress PS film and micro porous silicon template or nano porous anodic alumina template for $10 \mathrm{~min}$, which allowed the PS melt to infiltrate into the pores of templates. Then, the moulds were cooled to around $80^{\circ} \mathrm{C}$ and the formed PS micropillars-aligned pattern was separated from micro porous silicon template by machine tension method. For the porous anodic alumina template, the formed PS nanopillars-aligned pattern were released by removing the template in $\mathrm{NaOH}$ solutions and rinsed thoroughly with deionized water and ethanol. 
The samples were dried in an oven at $30^{\circ} \mathrm{C}$ for 2 days $(0.02 \mathrm{MPa})$.

\subsection{Plasma treatment of PS micro/nano pillars-aligned patterns}

The PS micro/nano pillars-aligned patterns and PS original surface were treated using an air plasma instrument (PDC-32G, Harrick Plasma, USA). The input power is $100 \mathrm{~W}(220 \mathrm{~V})$ and a maximum of $18 \mathrm{~W}$ is applied to the RF coil with no RF emission. The frequency is $50 \mathrm{~Hz}$. The vacuum of $20 \mathrm{~Pa}$ was employed during the whole irradiation process. The distance from the sample to the top of chamber ( $7.62 \mathrm{~cm}$ diameter) is about $3.8 \mathrm{~cm}$. Plasma irradiation time for all the specimens was set at $90 \mathrm{sec}-$ onds. As comparison, the PS coating on silicon wafer was also prepared with its tetrahydrofuran solution $(0.5 \mathrm{~g} / \mathrm{ml})$ on a Spin Coater (CEE model 100CB, Brewer Science Co., USA). The sample

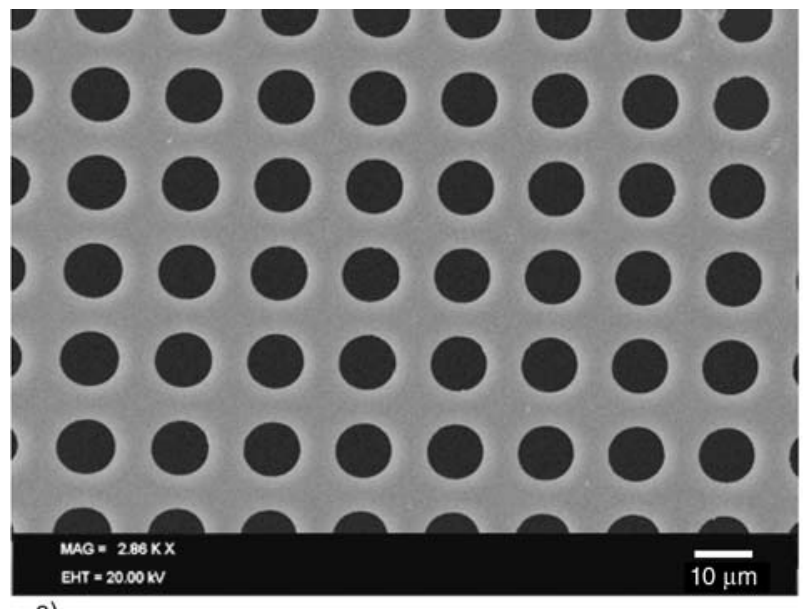

a)

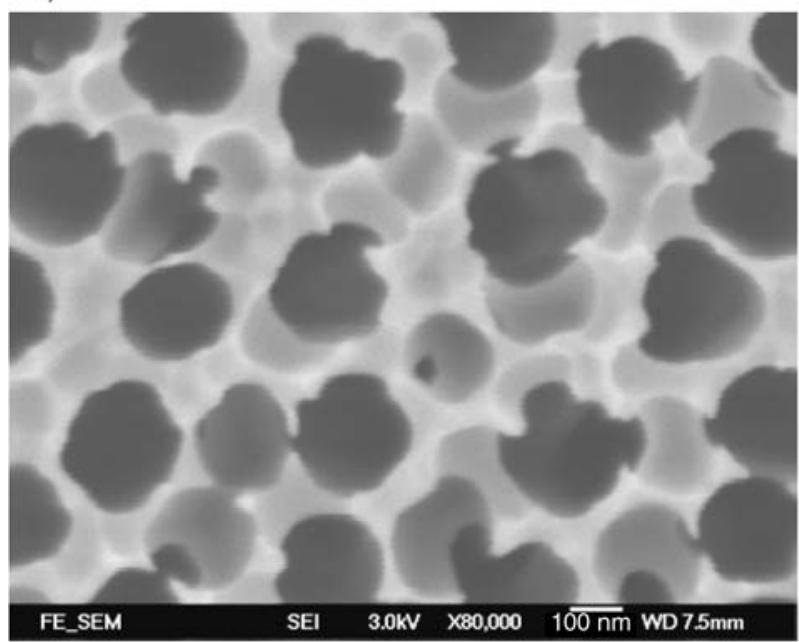

c) was dry in the oven at $30^{\circ} \mathrm{C}$ for 2 days $(0.02 \mathrm{MPa})$. The plasma irradiation process is same as micro/ nano pillars-aligned patterns.

\subsection{Characterization and measurement}

Micro porous silicon template, nano PAA template and the as-prepared PS micro/nano pillars-aligned patterns were characterized using scanning electron microscopy (SEM, Leica Stereoscan 440, Leica Co., Germany) and field-emission scanning electron microscopy (FE-SEM, JEOL JSM-6335F, JEOL Ltd., Japan). All samples were coated with $5 \mathrm{~nm}$ Au before measurements.

Water static CA was measured using a contact angle goniometer (Model 250-F1, Rame-hart Instrument Co., USA) at ambient temperature. A drop of ion-exchanged water $(3 \mu \mathrm{l})$ was placed on the asprepared surface using microsyringe appurtenance, then a photograph was taken with drop image

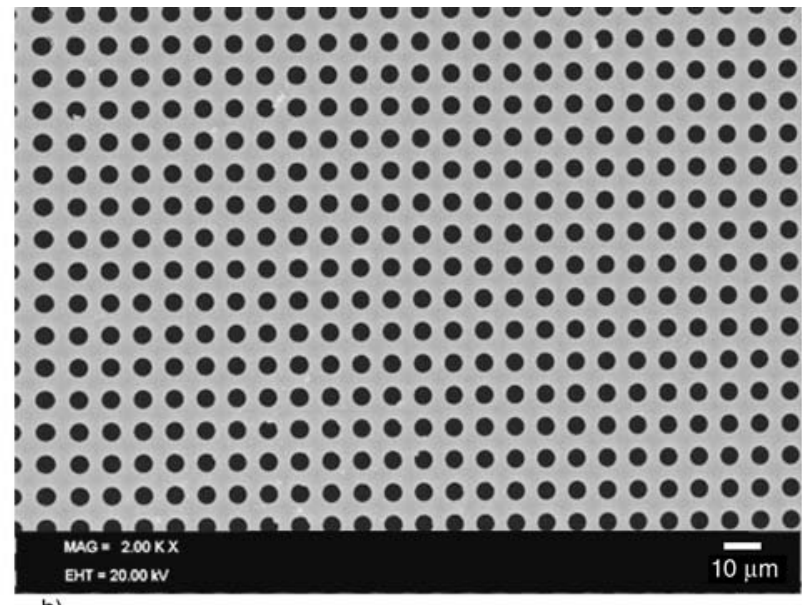

b)

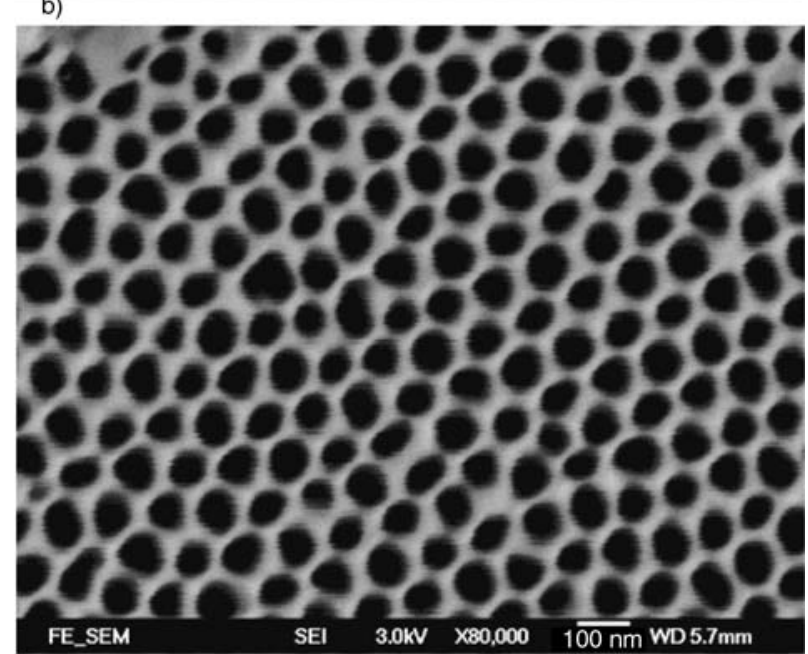

d)

Figure 1. SEM images of micro and nano porous templates, (a) silicon template with pore diameter of $10 \mu \mathrm{m}$; (b) silicon template with pore diameter of $5 \mu \mathrm{m}$; (c) alumina template with pore diameter of $250 \mathrm{~nm}$; (d) alumina template with pore diameter of $100 \mathrm{~nm}$ 
Advanced 2.1 software. The contact angle was determined by fitting a Young-Laplace curve around the drop. Six parallel measurements were made for each sample, and the average contact angle was taken as the result for each surface.

\section{Results and discussion}

\subsection{PS micro/nano pillars-aligned patterns}

The microporous silicon templates were fabricated by combined process of UV lithography and ICP etching [30], and the nanoporous anodic alumina templates were prepared by the so-called two-step anodic oxidation [31,32]. Their SEM top-view images are presented in Figure 1. In addition, the SEM cross-section images of a microporous silicon template and nanoporous anodic alumina template are shown in Figure 2.

The regular micropores with diameters of 5, 10 and $20 \mu \mathrm{m}$ were well prepared using the combined process of UV lithography and ICP etching. The diameters of micropores are uniform with the interval space of 3, 6 and $12 \mu \mathrm{m}$, respectively. The depth of all the micropores is about $20 \mu \mathrm{m}$ from its crosssection image (Figure 2a). For anodic alumina templates, average diameters of nanoporous are 100 and $250 \mathrm{~nm}$, respectively. And their depth of the two templates is about $50 \mu \mathrm{m}$ from the SEM crosssection observation (Figure $2 \mathrm{~b}$ ).

Employing these inorganic templates with high surface energy, the wetting and infiltration of PS melts into micro-/nanopores can be accomplished, which are driven by capillary force due to the high surface energy of silicon and alumina $[35,36]$. When PS

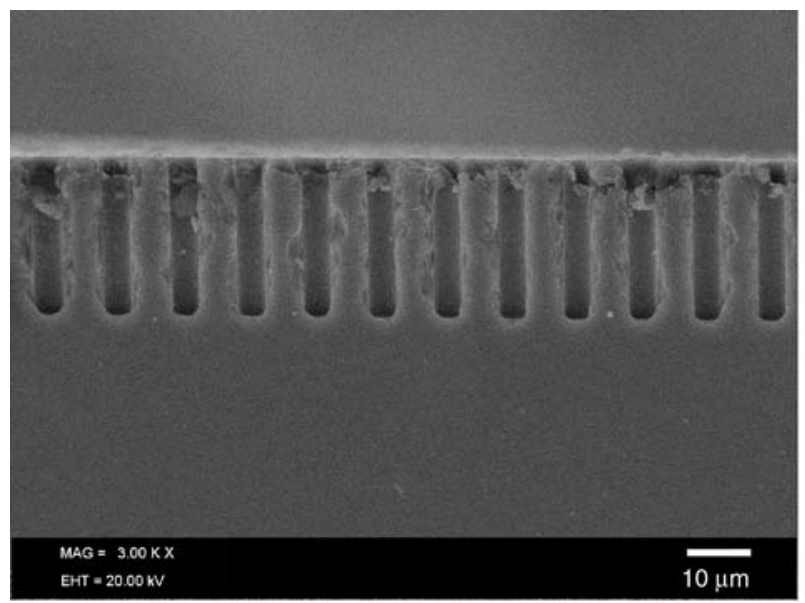

a) melts shrink after cooling to ambient temperature and templates are removed with mechanical demoulding method and $\mathrm{NaOH}$ solution, respectively. The generated PS one-dimensional micro/ nano pillars are aligned on the residual PS bulk substrate resulting in the formation of PS micro/nano pillars-aligned patterns as shown in Figure 3. The diameter of micropillars is nearly equal to the diameter of template employed. The height of micropillars from 10 and $20 \mu \mathrm{m}$ silicon templates is about $20 \mu \mathrm{m}$ as similar with their depth. However, due to the damage during the mechanical demoulding (Figure $3 \mathrm{~b}$ ), the height of micropillars from $5 \mu \mathrm{m}$ silicon template is only about $5 \mu \mathrm{m}$, so the aspect ratio is equal to that of micropillars from $20 \mu \mathrm{m}$ silicon template. On the other hand, the diameter of nanopillars with height of about $45 \mu \mathrm{m}$ (Figure 3e) is a little smaller than the diameter of template employed because the enhanced shrinkage effect on nanoscale after cooling from PS melts.

\subsection{Wettability transition from hydrophilicity to hydrophobicity of PS micro/nano pillars-aligned patterns}

To demonstrate the wettability transition behavior after the micro/nano patterning process and plasma irradiation, the static water CAs of the PS micro/ nano pillars-aligned patterns are presented in Figure 4, where the corresponding water droplet shapes are enclosed.

When the PS surface is patterned with aligned micropillars with diameters of 5, 10 and $20 \mu \mathrm{m}$, the static water CAs without plasma treatment are

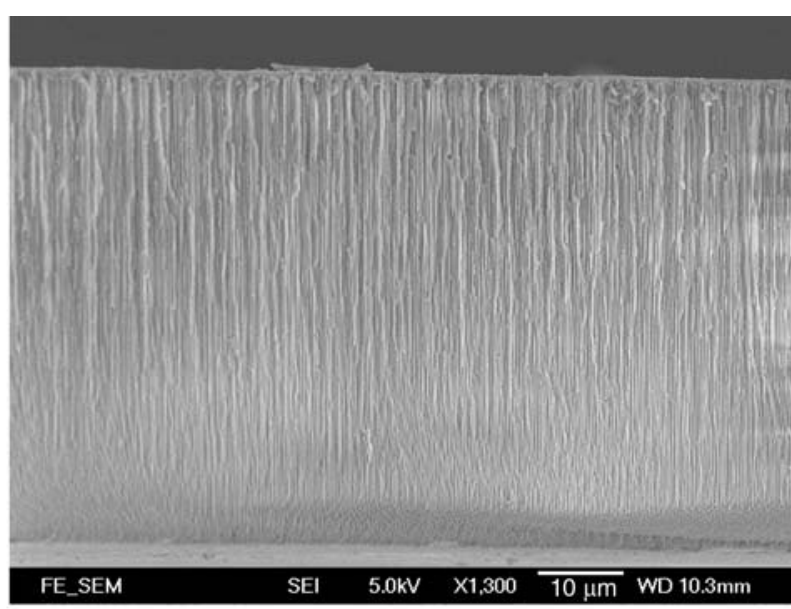

b)

Figure 2. SEM images of micro and nano porous templates, (a) cross-section of silicon template with pore diameter of $5 \mu \mathrm{m}$; (b) cross-section of alumina template with pore diameter of $250 \mathrm{~nm}$ 

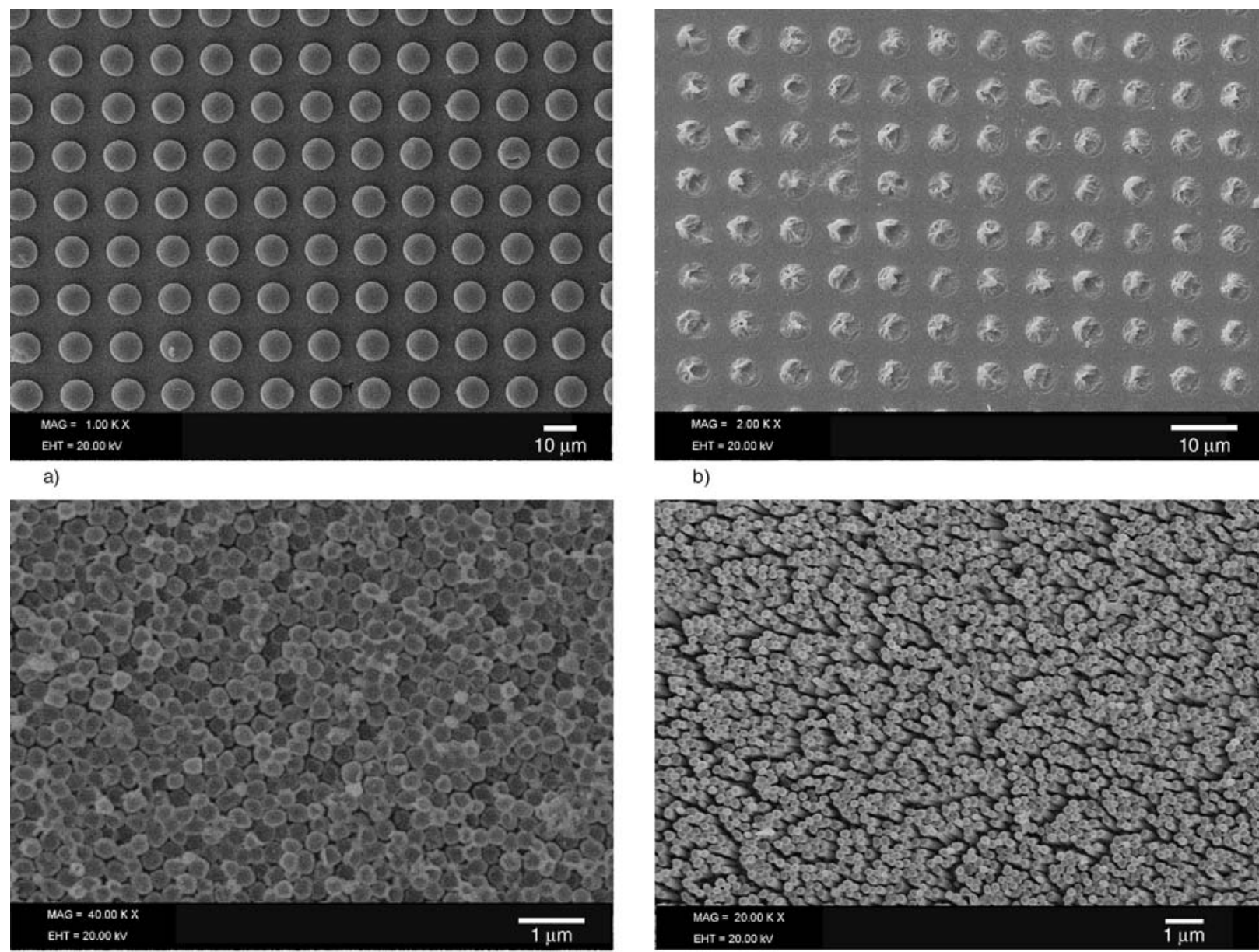

c)

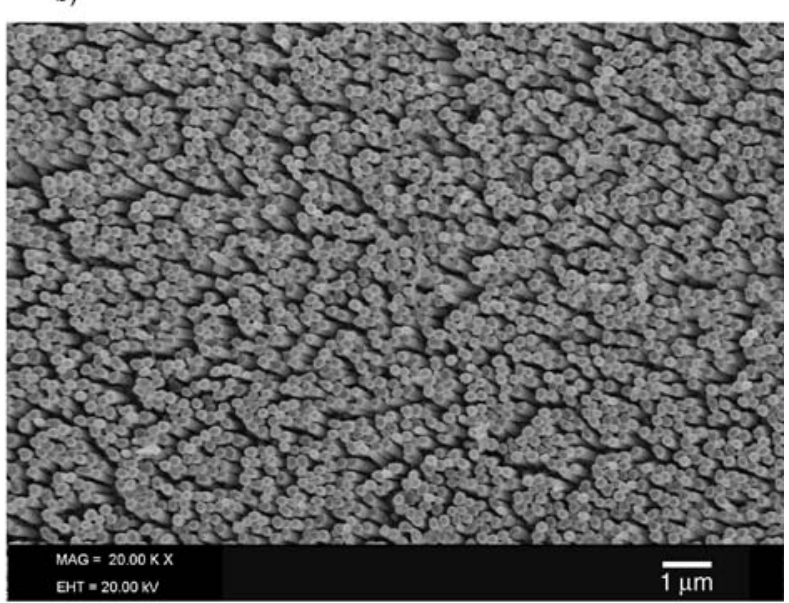

d)

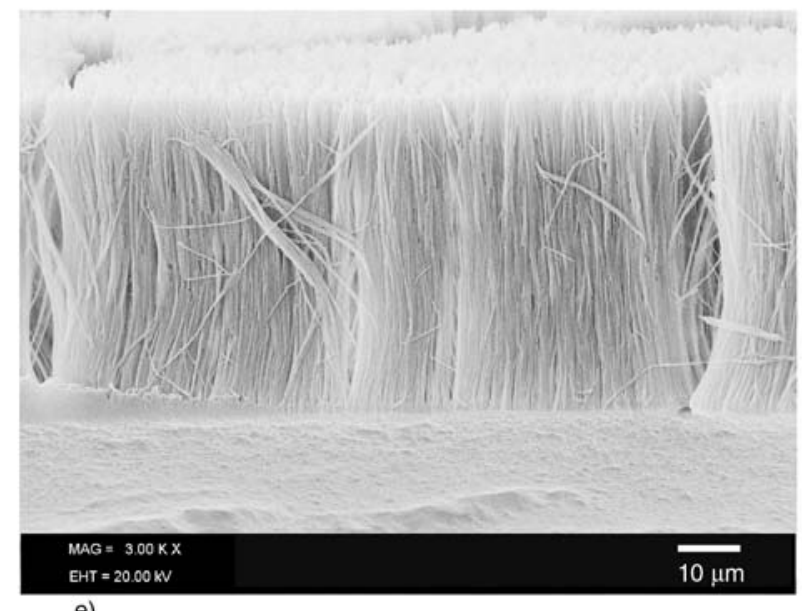

Figure 3. SEM images of PS micro/nano pillars-aligned patterns formed with aligned pillars with diameter of (a) $10 \mu \mathrm{m}$; (b) $5 \mu \mathrm{m}$; (c) $250 \mathrm{~nm}$; (d) $100 \mathrm{~nm}$; (e) cross-section of d

enhanced from $76^{\circ}$ (PS original surface) to $101^{\circ}$ that exhibits hydrophobicity. Similarly, when PS surface pattern is formed by the aligned nanopillars with diameter of 100 or $250 \mathrm{~nm}$, the static water CAs are increased to $130^{\circ}$ that exhibits high hydrophobicity. In a wide scale of pillar diameters from nano to micro, the wettabilities of PS micro/nano pillars- aligned patterns are changed from hydrophilicity to hydrophobicity after micro/nano patterning process. It is not surprizing because the PS surfaces are turned to be compounded rough surfaces consisting of micro/nano pillars and air [37, 38]. It is well known that the relationship between a compounded rough surface $\left(\theta_{c}\right)$ and the equilibrium contact 
angle on a flat surface $\left(\theta_{e}\right)$ can be described as the Cassie's equation (no-wetted state) [39] (Equation (1)):

$\cos \theta_{c}=\Phi_{s} \cos \theta_{e}-\left(1-\Phi_{s}\right)$

where $\Phi_{s}$ is the solid-liquid contact (or solid) fraction of the surface. Compared with flat surface, the PS micro/nano pillars-aligned patterns possess the greater contact area fraction of water droplet and air on the tip of pillars and their interval on patterns, so the hydrophobicity phenomena are observed.

\subsection{Wettability transition from hydrophobicity to hydrophilicity of PS micro/nano pillars-aligned patterns with plasma irradiation}

The wettability transition from hydrophilicity to hydrophobicity of PS micro/nano pillars-aligned patterns mentioned above is not surprizing, which can be also observed on the other polymer nanopatterned surfaces, such as the polyethylene nanopatterns or self-assembled fluorosilanated monolayer

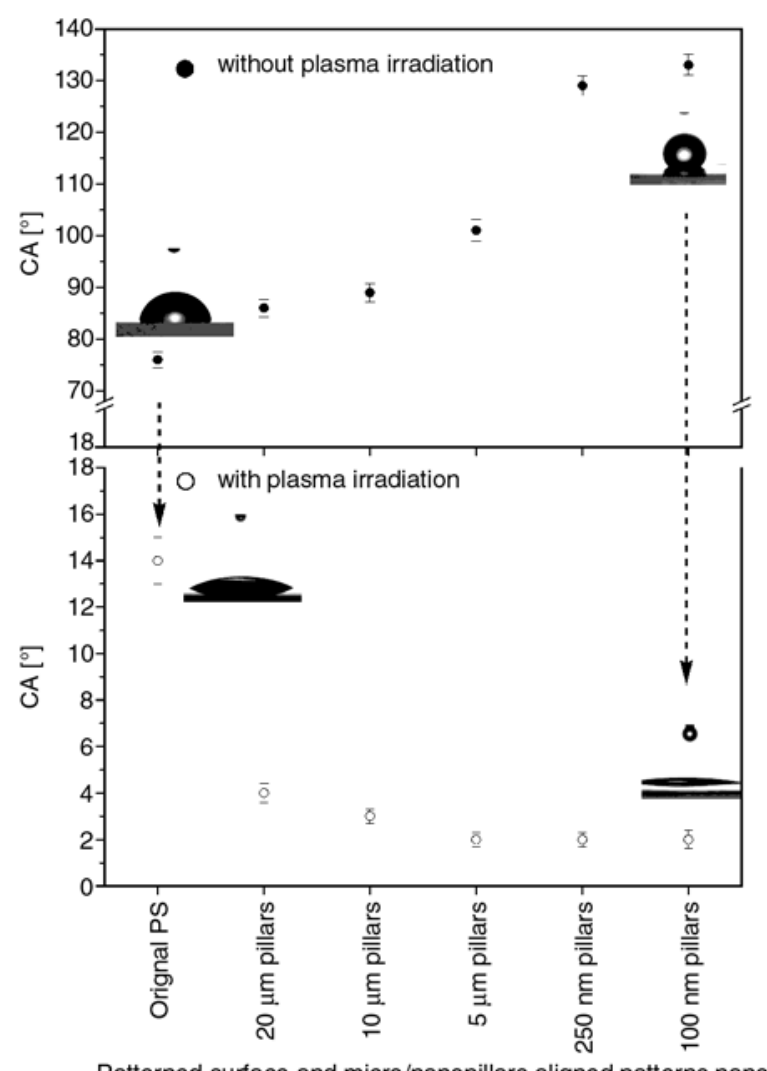

Figure 4. Static water contact angles of PS flat surface, micro/nano pillars-aligned patterns with and without plasma irradiation enclosed with the corresponding water droplet shape structures [29, 39]. However, after only 90 seconds air plasma irradiation $(200 \mathrm{~Pa})$, the interesting sharp transition from hydrophobicity to hydrophilicity of PS micro/nano pillars-aligned patterns was observed as presented in Figure 4.

After plasma irradiation, the PS original surface with weak hydrophilicity (CA of $76^{\circ}$ ) was transformed into a hydrophilic surface with CA of $15^{\circ}$. The PS coating on silicon wafer also show the similar transition (from 78 to $17^{\circ}$ ). However, the PS micro/nano pillars-aligned patterns were directly transformed from hydrophobic (CA of 101 and $130^{\circ}$, respectively) to superhydrophilic (CA less than $5^{\circ}$ ). That is, the PS micro/nano pillars-aligned patterns present sharp wettability transition from hydrophobicity to superhydrophilicity (from $130^{\circ}$ to less than $5^{\circ}$ ) compared with the flat PS surface (from 76 to $15^{\circ}$ ).

For PS surface with CA of $76^{\circ}$, a rough surface of micro/nano pillars-aligned patterns may make it hydrophobic according to Cassie state (no-wetted state). It is well known that plasma irradiation change the chemical composition of PS surface [40-42] to lead to a slow chain scission with hydroxyl group, carboxyl group, are discussed in references in detail [26, 43, 44]. If PS surface or coating was treated using air plasma irradiation, the hydrophilic surface with $\mathrm{CA}$ of $15^{\circ}$ was obtained. It indicates the water can wet PS surface according the Wenzel state (wet state). Under this condition, the water drop can diffuse along the contact surface on the tip of pillars and their interval on patterns during the static CA measurement. Compared with the PS original flat surface or coating, the high surface ratio of PS micro/nano pillars-aligned patterns enhance the diffuse effect thus resulting in the sharp transition from hydrophobicity to hydrophilicity. That is, the integrated effects of surface natural wettability and structure asperities lead to sharp transition from hydrophobicity to hydrophilicity of micro/nano pillars-aligned patterns with plasma irradiation.

\subsection{Wettability stabilities of PS micro/nano pillars-aligned patterns after plasma irradiation}

When plasma treated micro/nano pillars-aligned patterns are exposed to air, the wettability stability and durability of hydrophilicity is an important 


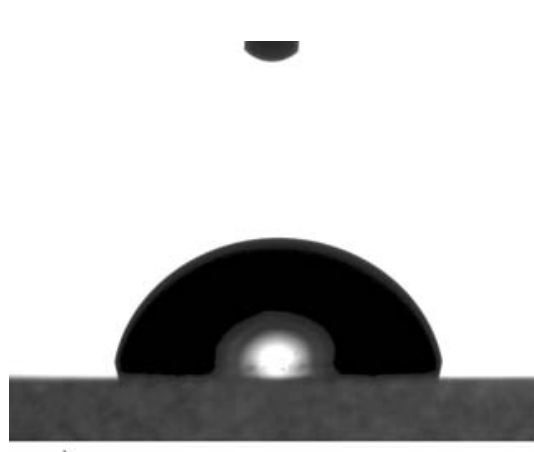

a)

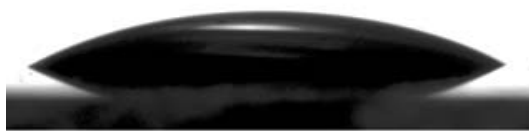

d)

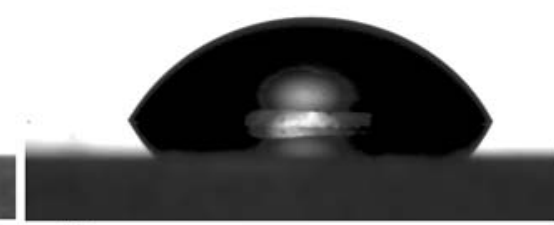

b)

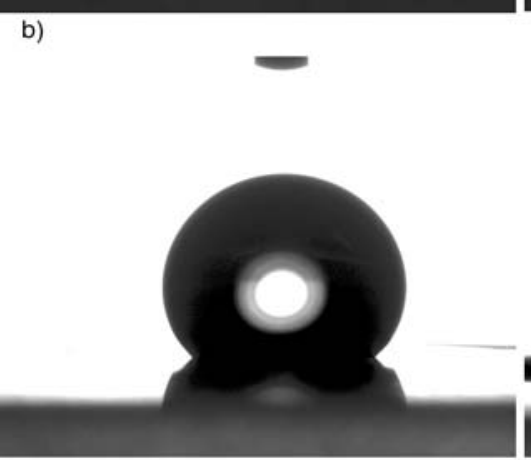

e)

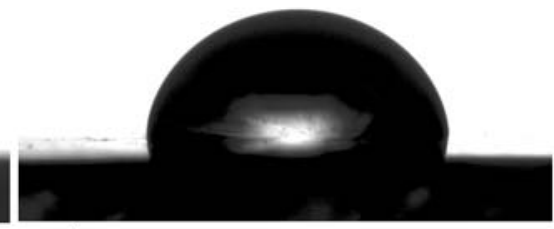

c)

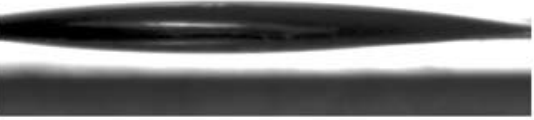

f)

Figure 5. Droplet shape on PS micro/nano pillars-aligned patterns, (a) PS flat surface; (b) plasma treated PS flat surface (after 2 days); (c) PS micro pillars-aligned patterns; (d) plasma treated PS micro pillars-aligned patterns (after 2 days); (e) PS nano pillars-aligned patterns; (f) plasma treated PS nano pillars-aligned patterns (after 2 days)

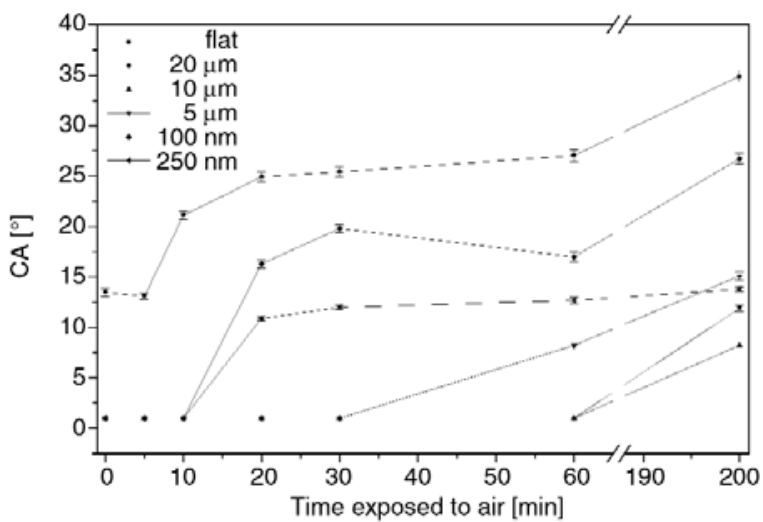

Figure 6. The development of water contact angles of PS micro/nano pillars-aligned patterns in a shortterm after plasma irradiation

issue. To investigate the wettability stability, a series of CA measurements were conducted as time going by. The droplet shapes are presented in Figure 5 and CA during the recovery process within the first $200 \mathrm{~min}$ and 2 days are shown in Figure 6 and Figure 7, respectively.

After being exposed to air for 2 days, the plasma treated PS micro/nano pillars-aligned patterns still exhibit obvious hydrophilicity $\left(15-32^{\circ}\right)$, while, the plasma treated PS flat surface recovers to CA of $45^{\circ}$. The recovery process in Figure 6 and 7 demonstrates that the hydrophilicity of micro/nano pillarsaligned patterns is more stable than that of PS flat

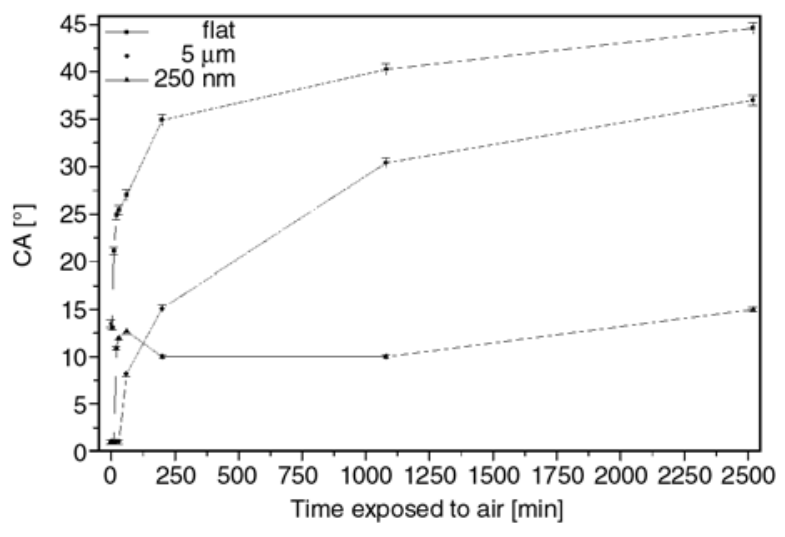

Figure 7. The development of water contact angles of PS micro/nano pillars-aligned patterns in a long term after plasma irradiation

surface no matter in $200 \mathrm{~min}$ recovery (short term) or in 2 days recovery (long term) after plasma irradiation. Because that chemistry change has time dependence, the hydrophilic polymer surface obtained using plasma irradiation can usually be recovered to its original wettabilty state to some degree just like the PS flat surface recovery. But for micro/nano pillars-aligned patterns with high surface ratio, the recovery is obvious lagging due to the dynamic contact angle hysteresis phenomena [39].

On the other hand, the recovery also demonstrates a little difference between micro and nano pillars- 
aligned patterns. After plasma irradiation, the nano pillars-aligned patterns keep on superhydrophilic state for $10 \mathrm{~min}$, then $\mathrm{CA}$ is increased to $15^{\circ}$ within 10 min. Comparatively, the micro pillars-aligned patterns keep on superhydrophilic state for 30 to $60 \mathrm{~min}$. The CA of micro pillars-aligned patterns is below that of nano pillars-aligned patterns within the first $200 \mathrm{~min}$. On the other hand, the recovery of the micro pillars-aligned patterns continue even after 2 days, while, the recovery for the nano pillars-aligned patterns turns weak after $230 \mathrm{~min}$. After $230 \mathrm{~min}$, the CA of the micro pillars-aligned patterns slowly exceeded that of nano pillarsaligned patterns. Since the nano pillars-aligned patterns possess higher high surface ratio than micro pillars-aligned patterns, nano pillars-aligned patterns exhibit higher stability in the long run than micro pillars-aligned patterns. The differences in the CA stability profiles between micro/nano pillars-aligned patterns and flat original surface are not only interesting, but also useful in providing new possible methods for creating programmable surfaces for particle capture and releasing in drug delivery and DNA analysis.

\section{Conclusions}

PS micro/nano pillars-aligned patterns were prepared using hot embossing on silicon microporous template and alumina nanoporous template. The combination of micro/nano patterning and plasma irradiation can easily regulate wettabilities of PS surfaces, i.e. from hydrophilicity to hydrophobicity, or from hydrophobicity to superhydrophilicity. When the wettability transition from hydrophobicity to hydrophilicity occurs, there is only mild hydrophilicity loss. And the hydrophilicity of PS micro/nano pillars-aligned patterns is more stable in 200 min and 2 days recovery than that of PS flat surfaces after plasma irradiation.

\section{Acknowledgements}

This work was financially supported with the Aero-Science Fund of China (2009ZH53073) and the National Natural Science Foundation of China (20874080), Research Grants Council of Hong Kong (RGC, PolyU5327/07E).

\section{References}

[1] Schulz B., Frommer W. B.: A plant ABC transporter takes the lotus seat. Science, 306, 622-625 (2004). DOI: $10.1126 /$ science.1105227

[2] Ma Y., Cao X. Y., Feng X. J., Ma Y. M., Zou H.: Fabrication of super-hydrophobic film from PMMA with intrinsic water contact angle below $90^{\circ}$. Polymer, 48 , 7455-7460 (2007).

DOI: 10.1016/j.polymer.2007.10.038

[4] Liu Q. T., Zhang H., Yin S. Y., Wu L. X., Shao C., Su Z. M.: Hierarchical self-assembling of dendritic-linear diblock complex based on hydrogen bonding. Polymer, 48, 3759-3770 (2007).

DOI: 10.1016/j.polymer.2007.04.041

[5] Zhang W., Chu P. K., Jia J. H., Zhang Y. H., Fu Ricky K. Y., Yan Q.: Antibacterial properties of plasmamodified and triclosan or bronopol coated polyethylene. Polymer, 47, 931-936 (2006).

DOI: 10.1016/j.polymer.2005.12.009

[6] Chen J-K., Chan C-H., Chang F-C.: Immobilization of layered double hydroxides in the fluidic system for nanoextraction of specific DNA molecules. Applied Physics Letters, 92, 053108/1-053108/3 (2008). DOI: $\underline{10.1063 / 1.2840175}$

[7] Ruiz A., Valsesia A., Ceccone G., Gilliland D., Colpo P., Rossi F.: Fabrication and characterization of plasma processed surfaces with tuned wettability. Langmuir, 23, 12984-12989 (2007). DOI: $\underline{10.1021 / 1 a 702424 \mathrm{r}}$

[8] Tsujii K., Yamamoto T., Onda T., Shibuichi S.: Super oil-repellent surfaces. Angewandte Chemie International Edition, 36, 1011-1012 (1997). DOI: 10.1002/anie.199710111

[9] Morita M., Koga T., Otsuka H., Takahara A.: Macroscopic-wetting anisotropy on the line-patterned surface of fluoroalkylsilane monolayers. Langmuir, 21, 911-918 (2005).

DOI: $\underline{10.1021 / 1 \mathrm{a} 0485172}$

[10] Puukilainen E., Rasilainen T., Suvanto M., Pakkanen T. A.: Superhydrophobic polyolefin surfaces: Controlled micro- and nanostructures. Langmuir, 23, 7263-7268 (2007).

DOI: $\underline{10.1021 / \mathrm{la} 063588 \mathrm{~h}}$

[11] Kojio K., Mitsui Y., Furukawa M.: Synthesis and properties of highly hydrophilic polyurethane based on diisocyanate with ether group. Polymer, 50, 3693 3697 (2009). DOI: 10.1016/j.polymer.2009.05.030

[12] Yi F. P., Zheng S. X.: Effect of hydrophobic polystyrene microphases on temperature-responsive behavior of poly ( $N$-isopropylacrylamide) hydrogels. Polymer, 50, 670-678 (2009). DOI: 10.1016/j.polymer.2008.11.038 
[13] Nurmi L., Holappa S., Nykänen A., Laine J., Ruokolainen J., Seppälä J.: Ultra-thin films of cationic amphiphilic poly(2-(dimethylamino)ethyl methacrylate) based block copolymers as surface wettability modifiers. Polymer, 50, 5250-5261 (2009).

DOI: $10.1016 /$ j.polymer.2009.08.043

[14] Wang Z., Koratkar N., Ci L., Ajayan P. M.: Combined micro-/nanoscale surface roughness for enhanced hydrophobic stability in carbon nanotube arrays. Applied Physics Letters, 90, 143117/1-143117/3 (2007).

DOI: $10.1063 / 1.2720761$

[15] Steinhart M., Wendorff J. H., Greiner A., Wehrspohn R. B., Nielsch K., Schilling J., Choi J., Gösele U.: Polymer nanotubes by wetting of ordered porous templates. Science, 296, 1997 (2002).

DOI: $\underline{10.1126 / \text { science. } 1071210}$

[16] Conrad P. G., Nishimura P. T., Aherne D., Schwartz B. J., Wu D., Fang N., Zhang X., Roberts M. J., Shea K. J.: Functional molecularly imprinted polymer microstructures fabricated using microstereolithography. Advanced Materials, 15, 1541-1544 (2003). DOI: $\underline{10.1002 / \mathrm{adma} .200304602}$

[17] Vogler M., Wiedenberg S., Mühlberger M., Bergmair I., Glinsner T., Schmidt H., Kley E. B., Grützner G.: Development of a novel, low-viscosity UV-curable polymer system for UV-nanoimprint lithography. Microelectronic Engineering, 84, 984-988 (2007). DOI: $10.1016 /$ j.mee.2007.01.184

[18] Mata A., Fleischman A. J., Roy S.: Fabrication of multi-layer SU-8 microstructures. Journal of Micromechanics and Microengineering, 16, 276-284 (2006). DOI: $10.1088 / 0960-1317 / 16 / 2 / 012$

[19] Guo C. W., Feng L., Zhai J., Wang G. J., Song Y. L., Jiang L., Zhu D. B.: Large-area fabrication of a nanostructure-induced hydrophobic surface from a hydrophilic polymer. ChemPhysChem, 5, 750-753 (2004). DOI: $\underline{10.1002 / \mathrm{cphc} .200400013}$

[20] Liu H., Li S. H., Zhai J., Li H. J., Jiang L., Zhu D. B.: Self-assembly of large-scale micropatterns on aligned carbon nanotube films. Angewandte Chemie International Edition, 43, 1146-1149 (2004). DOI: $10.1002 /$ anie. 200351988

[21] Zhang L., Zhou Z., Cheng B., DeSimone J. M., Samulski E. T.: Superhydrophobic behavior of a perfluoropolyether lotus-leaf-like topography. Langmuir, 22, 8576-8580 (2006). DOI: $\underline{10.1021 / 1 a 061400 \text { o }}$

[22] Lee J. A., McCarthy T. J.: Polymer surface modification: Topography effects leading to extreme wettability behavior. Macromolecules, 40, 3965-3969 (2007). DOI: $10.1021 / \mathrm{ma} 070061 \mathrm{i}$

[23] Feng L., Li S., Li Y., Li H., Zhang L., Zhai J., Song Y., Liu B., Jiang L., Zhu D.: Super-hydrophobic surfaces: From natural to artificial. Advanced Materials, 14, 1857-1860 (2002). DOI: $\underline{10.1002 / \mathrm{adma} .200290020}$
[24] Liu H., Zhai J., Jiang L.: Wetting and anti-wetting on aligned carbon nanotube films. Soft Matter, 2, 811821 (2006).

DOI: $10.1039 / \mathrm{b} 606654 \mathrm{~b}$

[25] Guruvenket S., Komath M., Vijayalakshmi S. P., Raichur A. M., Rao M. G.: Wettability enhancement of polystyrene with electron cyclotron resonance plasma with argon. Journal of Applied Polymer Science, 90, 1618-1623 (2003).

DOI: 10.1002/app.12816

[26] Chan C-M., Ko T-M., Hiraoka H.: Polymer surface modification by plasmas and photons. Surface Science Reports, 24, 1-54 (1996). DOI: $10.1016 / 0167-5729(96) 80003-3$

[27] Dhaya M., Parry K. L., Short R. D., Bradley J. W.: Investigating the plasma surface modification of polystyrene at low ion power densities. Journal of Physical Chemistry B, 108, 14000-14004 (2004).

DOI: $10.1021 / j p 0477046$

[28] Dhayal M., Forder D., Short R. D., Bradley J. W.: Electron temperature control in low-pressure plasmas using a two-mesh-separation technique. Vacuum, 70, 67-71 (2003). DOI: $\underline{10.1016 / \mathrm{S} 0042-207 X(02) 00769-8}$

[29] Kong J., Yung K-L., Xu Y., He L., Lau K. H., Chan C. Y.: Self-organized micropatterns of high aspect ratio polymer nanofibers by wetting of nanopores. Journal of Polymer Science Part B: Polymer Physics, 46, 1280-1289 (2008). DOI: $10.1002 /$ polb.21462

[30] Wang X. D., Chen Y. F., Wang L., Cui Z.: Fabrication of nanoimprint template in $\mathrm{Si}$ with high etch rate by non-switch DRIE process. Microelectronic Engineering, 85, 1015-1017 (2008).

DOI: $\underline{10.1016 / \mathrm{j} . \mathrm{mee} .2008 .01 .073}$

[31] Masuda H., Fukuda K.: Ordered metal nanohole arrays made by a 2-step replication of honeycomb structures of anodic alumina. Science, 268, 14661468 (1995). DOI: $10.1126 /$ science.268.5216.1466

[32] Xu T. T., Piner R. D., Ruoff R. S.: An improved method to strip aluminum from porous anodic alumina films. Langmuir, 19, 1443-1445 (2003). DOI: $\underline{10.1021 / \mathrm{la} 0264724}$

[33] Yung K-L., Kong J., Xu Y.: Studies on flow behaviors of polymer melts in nanochannels by wetting actions. Polymer, 48, 7645-7652 (2007). DOI: $10.1016 /$ j.polymer.2007.11.013

[34] Kong J., Xu Y., Yung K. L., Xie Y. C., He L.: Enhanced polymer melts flow though nanoscale channels under vibration. The Journal of Physical Chemistry C, 113, 624-629 (2009). DOI: $10.1021 / \mathrm{jp} 809164 \mathrm{k}$

[35] Moon S. I., McCarthy T. J.: Template synthesis and self-assembly of nanoscopic polymer 'Pencils'. Macromolecules, 36, 4253-4255 (2003). DOI: $\underline{10.1021 / \mathrm{ma} 0300239}$ 
[36] Zhang M. F., Dobriyal P., Chen J-T., Russell T. P.: Wetting transition in cylindrical alumina nanopores with polymer melts. Nano Letters, 6, 1075-1079 (2006).

DOI: $\underline{10.1021 / \mathrm{nl} 060407 \mathrm{n}}$

[37] Feng X. J., Jiang L.: Design and creation of superwetting/antiwetting surfaces. Advanced Materials, 18, 3063-3078 (2006).

DOI: $10.1002 / \mathrm{adma} .200501961$

[38] Jin M. H., Feng X. J., Feng L., Sun T. L., Zhai J., Li T. J., Jiang L.: Superhydrophobic aligned polystyrene nanotube films with high adhesive force. Advanced Materials, 17, 1977-1981 (2005). DOI: $10.1002 / \mathrm{adma} .200401726$

[39] Yeh K-Y., Chen L-J., Chang J-Y.: Contact angle hysteresis on regular pillar-like hydrophobic surfaces. Langmuir, 24, 245-251 (2008).

DOI: $10.1021 / 1 \mathrm{la} 7020337$

[40] Lejeune M., Lacroix L. M., Brétagnol F., Valsesia A., Colpo P., Rossi F.: Plasma-based processes for surface wettability modification. Langmuir, 22, 3057-3061 (2006).

DOI: $\underline{10.1021 / \mathrm{la} 052515 \mathrm{f}}$
[41] Arpagaus C., Rossi A., von Rohr P. R.: Short-time plasma surface modification of HDPE powder in a Plasma Downer Reactor - Process, wettability improvement and ageing effects. Applied Surface Science, 252, 1581-1595 (2005).

DOI: 10.1016/j.apsusc.2005.02.099

[42] Sanchis R. M., Calvo O., Sánchez L., García D., Balart R.: Enhancement of wettability in low density polyethylene films using low pressure glow discharge $\mathrm{N}_{2}$ plasma. Journal of Polymer Science Part B: Polymer Physics, 45, 2390-2399 (2007).

DOI: $\underline{10.1002 / \text { polb. } 21246}$

[43] Idage S. B., Badrinarayanan S.: Surface modification of polystyrene using nitrogen plasma. An X-ray photoelectron spectroscopy study. Langmuir, 14, 2780 2785 (1998).

DOI: $10.1021 / \mathrm{la} 9711286$

[44] Di Mundo R., Palumbo F., d'Agostino R.: Nanotexturing of polystyrene surface in fluorocarbon plasmas: From sticky to slippery superhydrophobicity. Langmuir, 24, 5044-5051 (2008).

DOI: $10.1021 / 1 a 800059 a$ 